\title{
Impact of Different Methodologies on the Detection of Point Mutations in Routine Air-dried Fine Needle Aspiration (FNA) Smears
}

\author{
Authors \\ C. Rehfeld ${ }^{1 *}$, S. Münz ${ }^{1 *}$, A. Krogdahl ${ }^{2}$, E. M. P. Jensen ${ }^{2}$, U. Siebolts ${ }^{3}$, C. Ferraz ${ }^{1}$, E. Bösenberg ${ }^{1}$, \\ L. Hegedüs ${ }^{4}$, R. Paschke ${ }^{1}$, M. Eszlinger ${ }^{1}$ \\ Affiliations \\ Division of Endocrinology and Nephrology, University of Leipzig, Leipzig, Germany \\ Department of Pathology, Odense University Hospital, Odense, Denmark \\ Institute of Pathology, University Hospital of Leipzig, Leipzig, Germany \\ Department of Endocrinology and Metabolism, Odense University Hospital, Odense, Denmark
}

\author{
Key words \\ thyroid cancer \\ - diagnostics \\ mutations \\ - fine-needle aspiration \\ thyroid cytology
}

received 27.10.2012

accepted 18.02.2013

\section{Bibliography}

DOI http://dx.doi.org/

10.1055/s-0033-1337987

Published online:

March 18, 2013

Horm Metab Res 2013;

45: 513-517

(c) Georg Thieme Verlag KG

Stuttgart · New York

ISSN 0018-5043

\section{Correspondence}

\section{R. Paschke, MD}

Division of Endocrinology and Nephrology

University of Leipzig

Liebigstrasse 20

04103 Leipzig

Germany

Tel.: + 49/341/9713 201

Fax: +49/341/9713 209

pasr@medizin.uni-leipzig.de

\section{Abstract}

$\nabla$

Currently the best method to select suspicious thyroid nodules for surgery is fine needle aspiration (FNA) cytology. However, FNA cytology has some inherent limitations, which can partly be overcome by molecular analysis. Therefore, molecular testing for somatic mutations has emerged as the most promising approach for molecular FNA diagnostics. The objective of this methodological study was to evaluate the feasibility of detecting BRAF, NRAS, HRAS, and $K R A S$ mutations from routine air-dried thyroid FNA smears, and to find an optimal method for detecting these mutations in FNA samples. DNA was extracted from 110 routine air-dried FNA smears and the corresponding surgically obtained formalin-fixed paraffin-embedded tissues. The presence of BRAF, NRAS, HRAS, and KRAS mutations was assessed by real-time PCRs and high resolution melting analysis, and/or pyrose-

\section{Introduction}

\section{$\nabla$}

Thyroid cancer comprises $94.5 \%$ of all endocrine malignancies, and ranks $13^{\text {th }}$ in frequency of occurrence among all cancers, accounting for $2.6 \%$ of all cases [1]. The majority of thyroid cancers are well-differentiated malignancies originating from thyroid follicular cells. The most frequent histotypes are papillary thyroid carcinomas (PTCs), followed by follicular thyroid carcinomas (FTCs). Currently the best method to select suspicious nodules for surgery is ultrasound-guided fine needle aspiration (FNA) cytology [2,3]. However, despite high specificity and sensitivity, FNA cytology has some inherent limitations, which can partly be overcome by molecular analysis, since RET/PTC rearrangements and $B R A F$ point mutations have been detected in

*These authors contributed equally to this work. quencing in comparison to real-time PCRs using hybridization probes and fluorescence melting curve analysis. The high-resolution melting-PCRs revealed a significantly lower number of PCR failures and questionable results, and detected more mutations than the PCRs using hybridization probes. The number of PCR failures ranging from $14-16 \%$ by high-resolution melting-PCRs could be further reduced to $5-14 \%$ by adding pyrosequencing assays. Moreover, pyrosequencing increased the specificity of the assays, up to $98-100 \%$, while the sensitivity ranged between $32-63 \%$. In summary, the mutation detection, especially in air-dried FNA samples, improves when using PCR assays in combination with high resolution melting analysis. Additional improvement can be obtained by subsequent pyrosequencing in comparison to previously described real-time PCRs using hybridization probes and fluorescence melting curve analysis.

13-43\% and $29-69 \%$ of PTCs, respectively [4]. In FTCs, PAX8/PPARG rearrangements and RAS mutations have been detected in $25-63 \%$ and 40-53\%, respectively [4]. Therefore, molecular testing for somatic mutations has emerged as the most promising approach for molecular FNA diagnosis [5-7]. It allows improved discrimination of the "follicular proliferation/indeterminate" and "suspicious" FNA categories, leading to reduced numbers of diagnostic thyroidectomies and false negative FNAs [8].

Hitherto a variety of molecular techniques have been used to detect these mutations. These comprise LightCycler PCR using hybridization probes, quantitative (q)PCR and post-PCR melting curve analysis, allele specific PCR, direct sequencing, restriction fragment polymorphism analysis, colorimetric assays, and nested PCR [9-15]. In archived FNA smears a similar sensitivity in the 
Table 1 Primers for the detection of BRAF (V600E), HRAS (codon 61), KRAS (codon 12/13), and NRAS (codon 61) point mutations by HRM-PCRs and pyrosequencing.

$\begin{array}{lll}\text { Primer } & \text { GenBank ID } & \text { Sequence } \\ \text { BRAF-HRM-F } & \text { NM_004333 } & \text { GGTGATTTTGGTCTAGCTACAG } \\ \text { BRAF-HRM-R } & \text { NM_004333 } & \text { GGCCAAAAATTTAATCAGTGGA } \\ \text { KRAS-HRM-F } & \text { NM_033360 } & \text { AGGCCTGCTGAAAATGACTG } \\ \text { KRAS-HRM-R } & \text { NM_033360 } & \text { GCTGTATCGTCAAGGCACTCT } \\ \text { NRAS-HRM-F } & \text { NM_002524 } & \text { TGGTGAAACCTGTTTGTTGG } \\ \text { NRAS-HRM-R } & \text { NM_002524 } & \text { TCGCCTGTCCTCATGTATTG } \\ \text { HRAS-Pyro-F } & \text { NM_005343 } & \text { GGTCATTGATGGGGAGACGT } \\ \text { HRAS-Pyro-R } & \text { NM_005343 } & \text { Biotin-GCATGGCGCTGTACTCCT } \\ \text { HRAS-Pyro } & \text { NM_005343 } & \text { TCCTGGATACCGCCG }\end{array}$

detection of BRAF mutations has been shown for allele specific PCR, direct sequencing, colorimetric assay, and LightCycler PCR using hybridization probes $[8,11]$.

The objective of this methodological study was 1) to evaluate the feasibility of detecting $B R A F, N R A S, H R A S$, and KRAS point mutations from routine air-dried FNA smears from thyroid nodules, and 2) to find the optimal method for detecting these point mutations in FNA samples, starting with a well-described LightCycler PCR using hybridization probes (hybridization probePCR) [16]. We demonstrate that the mutation detection, especially in our air-dried FNA samples, improves when using a qPCR assay in combination with high-resolution melting (HRMPCR) analysis, and show a further improvement by subsequent pyrosequencing in comparison to the previously described hybridization probe-PCR [16].

\section{Materials and Methods}

$\nabla$

\section{Patients and samples}

In total, 110 routine air-dried FNA slides from patients who subsequently underwent surgery for thyroid nodules at the Odense University Hospital (Odense, Denmark) were retrospectively included into this study. Additionally, 110 corresponding formalin-fixed paraffin embedded (FFPE) slices were analyzed. All FNA samples were graded according to the ATA 2006 guidelines [17] by an experienced pathologist (A.K.). Overall, cytological evaluation of the FNA slides revealed 27 malignant, 67 indeterminate, 14 benign, and 2 nondiagnostic samples. Histological evaluation of the corresponding FFPE samples revealed 66 follicular adenomas (FAs), 13 FTCs, 24 PTCs, and 7 goiters. The study was approved by, and conducted according to the regulations of the Danish scientific ethics committees.

\section{Nucleic acid extraction from FNA smears}

DNA was extracted from the routine air-dried FNA smears by extending the recently published RNA extraction protocol [18]. Three hundred $\mu \mathrm{l}$ of $96 \% \mathrm{EtOH}$ was added to the lower phase of the phenol/chloroform extraction and the tube was gently mixed and centrifuged at $8000 \times g$ for $3 \mathrm{~min}$. Afterwards, the supernatant was removed and the pellet was incubated with sodium citrate solution for $30 \mathrm{~min}$. The tube was centrifuged at $8000 \times \mathrm{g}$ for $3 \mathrm{~min}$ and again incubated with sodium citrate solution for an additional $30 \mathrm{~min}$. After further centrifugation at $8000 \times \mathrm{g}$ for $3 \mathrm{~min}$ at room temperature, the pellet was washed with $70 \%$ EtOH. Following a further centrifugation, the pellet was dried at room temperature for $15 \mathrm{~min}$ and then resuspended in $50 \mu \mathrm{l}$ of TE buffer.
After freezing the DNA for $24 \mathrm{~h}$ it was thawed, vortexed, and centrifuged at $12000 \times \mathrm{g}$ for $1 \mathrm{~min}$. The supernatant containing the DNA was then transferred to a new tube.

\section{Nucleic acid extraction from FFPE slides}

DNA was extracted from the FFPE slides by extending the RNA extraction protocol [18]. While the flow through of the gDNA elimination spin column was used for RNA extraction according to the miRNeasy FFPE kit protocol, $200 \mu \mathrm{l}$ TE buffer was added to the column and incubated at room temperature for $5 \mathrm{~min}$. Subsequently, the column was centrifuged at $8000 \times g$ for $1 \mathrm{~min}$. Six hundred $\mu \mathrm{l}$ of $96 \% \mathrm{EtOH}$ and $30 \mu \mathrm{l}$ of aqueous sodium acetate were added, mixed, and incubated for $10 \mathrm{~min}$ at room temperature. After centrifugation for $10 \mathrm{~min}$ at room temperature the DNA pellet was washed with $70 \% \mathrm{EtOH}$ and eluted with $50 \mu \mathrm{l} \mathrm{TE}$ buffer.

\section{Detection of point mutations by hybridization probe- PCRs}

DNA extracted from the FNA samples was screened for point mutations in BRAF codons 600/601 and for point mutations in HRAS codon 61, KRAS codons 12/13, and NRAS codon 61 by realtime PCRs using hybridization probes and fluorescence melting curve analysis on a Lightcycler 480 according to Nikiforov et al. [16]. The PCRs for the detection of these point mutations (with exception of the PCR detecting point mutations in HRAS) were applicable to our DNA samples, which are (due to the extraction from routine FNA samples) of lower quality than the DNAs extracted from fresh FNA material.

Samples tested positive were subsequently analyzed by pyrosequencing on a PyroMark Q24 (QIAGEN).

\section{Detection of point mutations by PCRs using high resolution melting (HRM) analysis}

$B R A F, K R A S$, and NRAS point mutations were detected by realtime PCR and HRM (HRM-PCR) using primers flanking the mutation hotspots ( $\triangle$ Table 1) and the LightCycler 480 High Resolution Melting Master chemistry (Roche, Mannheim, Germany) on a LightCycler 480 (Roche, Mannheim, Germany) amplifying DNA fragments of less than $200 \mathrm{bp}$. Although several primer pairs were tested to establish a HRM-PCR to detect point mutations in codon 61 of HRAS, none of the PCRs gave reliable results. PCRs were processed through an initial denaturation at $95^{\circ} \mathrm{C}$ for 10 min followed by 55 cycles of a 3-step PCR, including $3 \mathrm{~s}$ of denaturation at $95^{\circ} \mathrm{C}$, a 12 -s annealing phase at $58^{\circ} \mathrm{C}(N R A S$, $K R A S) / 60^{\circ} \mathrm{C}(B R A F)$, and an elongation phase at $72^{\circ} \mathrm{C}$ for $10 \mathrm{~s}$. Subsequently, a high-resolution melting curve was assessed from $75-95^{\circ} \mathrm{C}$ with an increase of $0.02^{\circ} \mathrm{C} / \mathrm{s}$ and 25 acquisitions per degree. DNA from patient specimens known to carry $B R A F$, KRAS, and NRAS point mutations were used as positive controls in each analysis. Samples tested positive were hereafter analyzed by pyrosequencing on a PyroMark Q24 (QIAGEN).

\section{Detection of point mutations by pyrosequencing}

Point mutations in BRAF, KRAS, and NRAS were detected by pyrosequencing using the therascreen BRAF Pyro Kit, therascreen NRAS Pyro Kit, and PyroMark KRAS Kit (all from QIAGEN, Hilden, Germany) according to the manufacturer's instructions. In brief, $5 \mu$ l of genomic DNA was amplified using template specific PCR primers (including one biotin-labeled primer) and template specific PCR conditions. Afterwards, the PCR products were immobilized to streptavidin sepharose beads and single 


\section{BRAF}

Positive in mutation screening

Wild-type in mutation screening

Questionable result

No PCR product/low efficiency PCR

Chi-square $\mathrm{p}$-value

KRAS

Positive in mutation screening

Wild-type in mutation screening

Questionable result

No PCR product/low efficiency PCR

Chi-square $\mathrm{p}$-value

NRAS

Positive in mutation screening

Wild-type in mutation screening

Questionable result

No PCR product/low efficiency PCR

Chi-square $\mathrm{p}$-value
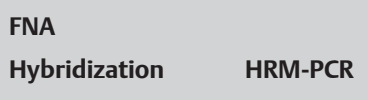

probe-PCR

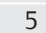

14

14

10

21

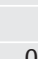

0

38

6

6

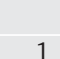

$1 \quad 10$

$43 \quad 38$

10

5

0.009495

\section{FFPE}

Hybridization HRM-PCR

probe-PCR

9
32
5
4

12

37

1

0

0.049

0
49
0
1
0.001499

0

$50 \quad 50$

0

0

0.001499

$\begin{array}{lcc}0 & 14 & 10 \\ 38 & 35 & 39 \\ 0 & 1 & 1 \\ 2 & 0 & 0 \\ 0.009495 & & 0.7416\end{array}$

The number of samples positive in mutation screening, wild-type in mutation screening, the number of questionable results, and the number of samples that could not be analyzed (due to no PCR product/low efficiency PCR) were compared by Chi-square tests between the 2 methods applied (hybridization probe-PCR and HRM-PCR)

stranded DNA was prepared allowing subsequent annealing of the sequencing primer to the template DNA. Then, the primed single stranded DNA was released from the streptavidin surface and transferred to a PyroMark Q24 (QIAGEN, Hilden, Germany) for pyrosequencing. HRAS point mutations were detected by pyrosequencing using self-designed primers ( $\bullet$ Table 1 ) and the following PCR conditions: PCRs were processed through an initial denaturation at $95^{\circ} \mathrm{C}$ for $15 \mathrm{~min}$ followed by 45 cycles of a 3-step PCR, including 20-s of denaturation at $95^{\circ} \mathrm{C}$, a $30-\mathrm{S}$ annealing phase at $64^{\circ} \mathrm{C}$, and a 30 -s elongation phase at $72^{\circ} \mathrm{C}$, followed by a final 5 min extension phase at $72^{\circ} \mathrm{C}$. Then, the PCR products were treated as described above.

\section{Statistical analysis}

The PCR results obtained by real-time PCR, using hybridization probes, and by real-time PCR and HRM were compared by the Chi square test. Cases with a wild type result in FNA and FFPE were considered "true negatives", and cases with a positive mutation screening in both, FNA and FFPE were considered "true positives". Cases with a positive mutation screening in the FNA sample and a wild type result in the FFPE were considered "false positive" and cases with a wild-type screening in the FNA and a positive mutations screening in the FFPE were considered "false negatives".

\section{Results}

$\nabla$

The HRM-PCR assays were developed and optimized using pGEM-T vectors carrying wild-type or mutated sequences of $B R A F, K R A S$, and NRAS, respectively. Normal and mutant sequences were mixed in proportions to give the following levels of mutation: $0,12.5,25,50$, and $100 \%$. These samples were run together with wild-type FFPE samples in one HRM-PCR run to determine the limit of detection for the different HRM assays. In all HRM-PCRs the limit of detection was approximately $12.5 \%$ (data not shown). In pyrosequencing, samples showing a mutation level greater than $5 \%$ were deemed mutation positive.

\section{Comparison of detecting point mutations by} hybridization probe-PCRs and by HRM-PCRs

Point mutations in BRAF, KRAS, and NRAS were detected by hybridization probe-PCRs and by HRM-PCRs in a subset of 50 FNA smears and corresponding FFPE samples, using DNA obtained from the same extractions for both methods. The results of hybridization probe-PCR and HRM-PCR for the FNAs were rated as "positive in mutation screening", "wild-type in mutation screening", “questionable result", or "no PCR product/ low efficiency PCR" ( $\diamond$ Table 2). While there are few or no significant differences between the hybridization probe-PCR and HRM-PCR for FFPE samples, the comparison of the 2 methods gave significant differences for the FNA samples ( $\bullet$ Table 2 ). For all 3 PCRs, HRM-PCR revealed a significantly lower number of questionable results, PCRs with no product, or low PCR efficiency, than the hybridization probe-PCR. For example, while the BRAF-hybridization probe-PCR revealed 31 questionable results/PCRs with no product or low PCR efficiency, only one BRAF-HRM-PCR revealed no product or low PCR efficiency ( $\triangle$ Table 2). Moreover, more mutations were detected by HRM than by hybridization probe-PCR. While 9 samples were tested mutation positive in the BRAF-HRM screening only 5 positive results were obtained by the hybridization probe-PCR ( $\bullet$ Table 2). While no KRAS mutation positive FNA sample could be identified by either hybridization probe based PCR or by HRM-PCR, 10 NRAS mutation positive samples were identified by HRMPCR, in comparison to 1 mutation positive sample by hybridization probe-PCR. Based on these results, the remaining 60 FNA and FFPE samples were analyzed by HRM-PCR only.

\section{Detection of point mutations by HRM-PCRs, HRM-PCRs and/or pyrosequencing in the total sample set of 110 FNA smears and corresponding FFPE samples}

First, all FFPE samples were screened for BRAF, KRAS, and NRAS mutations by HRM-PCRs. Positive and questionable HRM-PCR results were verified by pyrosequencing as were samples giving no PCR product in the HRM-PCR. Screening for HRAS mutations 
Table 3 Loss rate, sensitivity, specificity, and accuracy for detecting BRAF, HRAS, KRAS, and NRAS point mutations in 110 FNA smears compared to the corresponding FFPE samples.

\begin{tabular}{|c|c|c|}
\hline & $\begin{array}{l}\text { HRM-PCR } \\
\text { (percent) }\end{array}$ & $\begin{array}{l}\text { HRM-PCR combined } \\
\text { with PS (percent) }\end{array}$ \\
\hline \multicolumn{3}{|l|}{ BRAF } \\
\hline Loss rate & 16 & 5 \\
\hline Sensitivity & 54 & 63 \\
\hline Specificity & 97 & 98 \\
\hline Accuracy & 91 & 92 \\
\hline \multicolumn{3}{|l|}{ KRAS } \\
\hline Loss rate & 14 & 6 \\
\hline Sensitivity & NA & NA \\
\hline Specificity & 96 & 100 \\
\hline Accuracy & 96 & 100 \\
\hline \multicolumn{3}{|l|}{ NRAS } \\
\hline Loss rate & 14 & 12 \\
\hline Sensitivity & 47 & 32 \\
\hline Specificity & 95 & 99 \\
\hline Accuracy & 86 & 85 \\
\hline HRAS & \multicolumn{2}{|l|}{ PS (percent) } \\
\hline Loss rate & \multicolumn{2}{|l|}{14} \\
\hline Sensitivity & \multicolumn{2}{|l|}{40} \\
\hline Specificity & \multicolumn{2}{|l|}{100} \\
\hline Accuracy & \multicolumn{2}{|l|}{97} \\
\hline \multicolumn{3}{|c|}{$\begin{array}{l}\text { HRM-PCRs in combination with pyrosequencing (PS). HRAS point mutations were } \\
\text { detected by pyrosequencing alone. NA: Not analyzable }\end{array}$} \\
\hline
\end{tabular}

was done by pyrosequencing alone. The results of these analyses were deemed as the gold standard for the comparison of the results obtained from the FNA smear screening.

As done for the FFPE samples, all FNA samples were screened initially by HRM-PCRs (BRAF, KRAS, NRAS) or pyrosequencing (HRAS). For all mutation screenings, the loss rates (percentage of samples, which could not be analyzed due to the fact that the PCR revealed no PCR product or due to a low efficient PCR) were comparable. While $16 \%$ of FNA samples could not be analyzed in the BRAF-HRM-PCR, $14 \%$ of FNA samples could not be analyzed by either KRAS- or NRAS-HRM-PCR ( $\bullet$ Table 3 ). To reduce these loss rates all samples characterized by a less efficient PCR or no PCR product during the HRM-PCR screening were subsequently analyzed by pyrosequencing. In FNA smears, this procedure allowed a reduction of the loss rate to $5 \%$ in case of $B R A F$ mutation screening, $12 \%$ in case of NRAS mutation screening, and to $6 \%$ in KRAS mutation screening.

Although the HRM-PCR-screening of all mutations tested is characterized by a high specificity, ranging from $95 \%$ (KRAS) to $100 \%$ (HRAS), the combination with pyrosequencing further increased the specificity ( $\bullet$ Table 3 ). In contrast to the high specificity of the assays, the sensitivity of the FNA smear mutation screening was rather low, ranging from $32 \%$ (NRAS, HRM-PCR with pyrosequencing) to $63 \%$ (BRAF, HRM-PCR with pyrosequencing). Since no KRAS mutations could be detected a sensitivity for this assay cannot be calculated.

\section{Discussion}

$\nabla$

With the discovery of rearrangements (RET/PTC and PAX8/PPARG) and point mutations in the BRAF and several RAS genes in PTC and FTC, respectively, much knowledge has been added in rela- tion to their frequency, detection method, and their applicability in medical practice. Although, at present, ultrasound-guided FNA is the most sensitive method to diagnose nodules suspected of malignancy [2,3], molecular analysis has been demonstrated to be an important additional technique in raising the sensitivity of this method, especially in case of indeterminate samples $[5,6,8,9]$.

Recently, we could demonstrate, for the first time, the possibility of detecting RET/PTC and PAX8/PPARG rearrangements in routine air-dried FNA smears and in the corresponding FFPE [18]. This progress is associated with a number of advantages for the patients and society alike [8]. The aim of the present study was to evaluate the previously described hybridization probe-PCR [16] for the detection of BRAF, NRAS, KRAS, and HRAS point mutations in DNA from routine air-dried FNA smears. This DNA is degraded and of lower quality than the DNA from fine needle wash-outs, used by Nikiforov et al. [16]. Jin et al. [11] previously compared the detection of BRAF mutations by evaluating 4 different methods, including direct sequencing, Colorimetric Assay Mutector, LightCycler PCR, using hybridization probes, and an allele-specific PCR, using LightCycler SYBR Green showed similar sensitivities for these 4 detection methods. However, a multitude of other variables, such as sample source, number of samples, method of extraction, and conservation of the material, are also of importance. Indeed, there was no significant difference in the initial screening results of 50 FFPE samples of our set, analyzed by the hybridization probe-PCR [16] in comparison to a HRM-PCR ( $\odot$ Table 2). However, the screening of the initial 50 FNA samples revealed completely different results. The screening of these revealed a loss rate of $10 \%$ for NRAS and $42 \%$ for $B R A F$ when analyzing the FNA samples by the hybridization probe-PCR ( $\odot$ Table 3) [16]. In contrast, analyzing these FNA samples by the HRM-PCR reduced the loss rate to $0 \%$ for NRAS and $K R A S$, and to $2 \%$ for BRAF. Moreover, the use of HRM-PCR allowed the detection of more mutations than did the hybridization probe-PCR, in case of BRAF and NRAS ( $\bullet$ Table 2). These results clearly show that 1 ) the source of material, and the method of extraction and conservation of the material influence the outcome of the screening assay, and 2) that assays showing a similar performance in one type of material, can perform very differently using another type of material. Therefore, with respect to our samples, all further air-dried FNA smear analyses are based on the HRM-PCR, which is superior to the hybridization probe-PCR in analyzing routine air-dried FNA samples.

Although we observed low loss rates in the initial sample set by HRM-PCR, the analysis of the total sample set $(n=110)$ resulted in loss rates of 14 (KRAS, NRAS) to $16 \%(B R A F)$. Therefore, to further reduce the loss rates and to verify the results of the HRM screening, we performed pyrosequencing of all samples, which showed a positive, a questionable, or no result in HRM-PCRs. This algorithm resulted in a reduction of the loss rates in case of $B R A F$ and KRAS down to $5 \%$ and $6 \%$, respectively, while the loss rate for the NRAS assay decreased only slightly from 14 to $12 \%$. On top of improving the loss rates in 2 screening assays, adding pyrosequencing increased specificity in all screening assays (0 Table 3). However, while the specificity of our mutation screening is very high, the sensitivity is rather low. One reason for these low sensitivities might be the assay itself. This can be seen especially for the NRAS screening, where the HRM-PCR alone has a sensitivity of $47 \%$, which decreased to $32 \%$ after adding the pyrosequencing assay. This means that in particular the commercial NRAS pyrosequencing assay lacks sensitivity when 
analyzing degraded DNA samples. On the other hand we were able to improve the sensitivity from $54 \%$ to $63 \%$ in the $B R A F$ screening by adding pyrosequencing. Another reason for the low sensitivities might be tumor heterogeneity. Recently a pyrosequencing approach has reported percentages of mutant BRAF alleles from 5.1-44.7\% [19]. Tumor heterogeneity has been reported for $R E T / P T C$ rearrangements by fluorescence in situ hybridization $[20,21]$. This implies that the mutation screening of the FFPE sample may reveal a positive result, while the mutation screening of the FNA sample is negative. We speculate that potential reasons for this discrepancy could be aspiration of a mutation negative subpopulation or dilution of mutation positive cells by mutation negative cells during the aspiration process, resulting in a lower sensitivity. Further reasons for the rather low sensitivities might be a higher DNA degradation in older slides, a small number of tumor specific cells carrying the mutation in the FNA smear or a high number of contaminating cells (which might dilute a positive result from the tumor cells). Interestingly, the negative screening results were not significantly correlated with age of the samples, low cellularity of the FNA smears, or the presence of other cells. Therefore, the focus of optimizing the point mutation detection in DNA samples from routine air-dried FNA smears should be on improving the assays, in particular the NRAS pyrosequencing assay, aiming for a higher sensitivity.

In conclusion, we have shown that the previously described hybridization probe-PCRs [16], and the novel HRM-PCRs gave similar results, when analyzing FFPE samples. However, when analyzing degraded DNA from routine air-dried FNA smears, the HRM-PCR (in combination with pyrosequencing) outperformed the hybridization probe-PCR. Therefore, for the mutation analysis of routine air-dried FNA smears, HRM-PCR analysis should be the method of choice.

\section{Acknowledgements}

$\nabla$

This research was supported by Centro Nacional de Pesquisa e Tecnologia do Brasil (CNPq), Brazil to Carolina Ferraz and by a Deutsche Forschungsgemeinschaft (DFG) (ES162/4-1) and a Deutsche Krebshilfe grant to Markus Eszlinger. Laszlo Hegedüs is supported by an unrestricted grant from the Novo Nordisk Foundation. Ralf Paschke is supported by the DFG and the Deutsche Krebshilfe.

\section{Conflict of Interest \\ $\nabla$}

The authors declare that they have no conflicts of interest in the authorship or publication of this contribution.

\footnotetext{
References

1 Jemal A, Bray F, Center MM, Ferlay J, Ward E, Forman D. Global cancer statistics. CA Cancer J Clin 2011; 61: 69-90

2 Hegedus L. Clinical practice. The thyroid nodule. N Engl J Med 2004; 351: 1764-1771

3 Paschke R, Hegedus L, Alexander E, Valcavi R, Papini E, Gharib H. Thyroid nodule guidelines: agreement, disagreement and need for future research. Nat Rev Endocrinol 2011; 7: 354-361
}

4 Kondo T, Ezzat S, Asa SL. Pathogenetic mechanisms in thyroid follicular-cell neoplasia. Nat Rev Cancer 2006; 6: 292-306

5 Eszlinger M, Paschke R. Molecular fine-needle aspiration biopsy diagnosis of thyroid nodules by tumor specific mutations and gene expression patterns. Mol Cell Endocrinol 2010; 322: 29-37

6 Moses W, Weng J, Sansano I, Peng M, Khanafshar E, Ljung BM, Duh $\mathrm{QY}$, Clark $\mathrm{OH}$, Kebebew E. Molecular testing for somatic mutations improves the accuracy of thyroid fine-needle aspiration biopsy. World J Surg 2010; 34: 2589-2594

7 Nikiforova MN, Nikiforov YE. Molecular diagnostics and predictors in thyroid cancer. Thyroid 2009; 19: 1351-1361

8 Ferraz C, Eszlinger M, Paschke R. Current state and future perspective of molecular diagnosis of fine-needle aspiration biopsy of thyroid nodules. J Clin Endocrinol Metab 2011; 96: 2016-2026

9 Cantara S, Capezzone M, Marchisotta S, Capuano S, Busonero G, Toti $P$, Di SA, Caruso G, Carli AF, Brilli L, Montanaro A, Pacini F. Impact of proto-oncogene mutation detection in cytological specimens from thyroid nodules improves the diagnostic accuracy of cytology. J Clin Endocrinol Metab 2010; 95: 1365-1369

10 Hayashida N, Namba H, Kumagai A, Hayashi T, Ohtsuru A, Ito M, Saenko VA, Maeda S, Kanematsu T, Yamashita S. A rapid and simple detection method for the BRAF(T1796A) mutation in fine-needle aspirated thyroid carcinoma cells. Thyroid 2004; 14: 910-915

11 Jin L, Sebo TJ, Nakamura N, Qian X, Oliveira A, Majerus JA, Johnson MR, Lloyd RV. BRAF mutation analysis in fine needle aspiration (FNA) cytology of the thyroid. Diagn Mol Pathol 2006; 15: 136-143

12 Kimura ET, Nikiforova MN, Zhu Z, Knauf JA, Nikiforov YE, Fagin JA. High prevalence of BRAF mutations in thyroid cancer: genetic evidence for constitutive activation of the RET/PTC-RAS-BRAF signaling pathway in papillary thyroid carcinoma. Cancer Res 2003; 63: 1454-1457

13 Nikiforova MN, Kimura ET, Gandhi M, Biddinger PW, Knauf JA, Basolo F, Zhu Z, Giannini R, Salvatore G, Fusco A, Santoro M, Fagin JA, Nikiforov $Y E$. BRAF mutations in thyroid tumors are restricted to papillary carcinomas and anaplastic or poorly differentiated carcinomas arising from papillary carcinomas. J Clin Endocrinol Metab 2003; 88: 5399-5404

14 Rowe LM, Bentz BM, Bentz JM. Utility of BRAF V600E mutation detection in cytologically indeterminate thyroid nodules. Cytojournal 2006; 3: 10

15 Sapio MR, Posca D, Troncone G, Pettinato G, Palombini L, Rossi G, Fenzi G, Vitale $M$. Detection of BRAF mutation in thyroid papillary carcinomas by mutant allele-specific PCR amplification (MASA). Eur J Endocrinol 2006; 154: 341-348

16 Nikiforov YE, Steward DL, Robinson-Smith TM, Haugen BR, Klopper JP, Zhu Z, Fagin JA, Falciglia M, Weber K, Nikiforova MN. Molecular Testing for Mutations in Improving the Fine Needle Aspiration Diagnosis of Thyroid Nodules. J Clin Endocrinol Metab 2009; 94: 2092-2098

17 Cooper DS, Doherty GM, Haugen BR, Kloos RT, Lee SL, Mandel SJ, Mazzaferri EL, Mclver B, Sherman SI, Tuttle RM. Management guidelines for patients with thyroid nodules and differentiated thyroid cancer. Thyroid 2006; 16: 109-142

18 Ferraz C, Rehfeld C, Krogdahl A, Precht Jensen EM, Bosenberg E, Narz $F$, Hegedus L, Paschke R, Eszlinger M. Detection of PAX8/PPARG and RET/PTC Rearrangements Is Feasible in Routine Air-Dried Fine Needle Aspiration Smears. Thyroid 2012; 22: 1025-1030

19 Guerra A, Sapio MR, Marotta V, Campanile E, Rossi S, Forno I, Fugazzola $L$, Budillon A, Moccia T, Fenzi G, Vitale M. The primary occurrence of $\mathrm{BRAF}(\mathrm{V} 600 \mathrm{E})$ is a rare clonal event in papillary thyroid carcinoma. J Clin Endocrinol Metab 2012; 97: 517-524

20 Unger K, Zitzelsberger H, Salvatore G, Santoro M, Bogdanova T, Braselmann H, Kastner P, Zurnadzhy L, Tronko N, Hutzler P, Thomas G. Heterogeneity in the distribution of RET/PTC rearrangements within individual post-Chernobyl papillary thyroid carcinomas. J Clin Endocrinol Metab 2004; 89: 4272-4279

21 Zhu Z, Ciampi R, Nikiforova MN, Gandhi M, Nikiforov YE. Prevalence of RET/PTC rearrangements in thyroid papillary carcinomas: effects of the detection methods and genetic heterogeneity. J Clin Endocrinol Metab 2006; 91: 3603-3610 